\title{
Agentes comunitários de saúde: percepção sobre os serviços de saúde relacionados à doença de Chagas
}

\section{Community health agents: perception about health services related to Chagas' disease}

Fernanda Cristina Santos Rodrigues ${ }^{1}$ (D), Izabella Cristina Alves de Souza' (D), Alexandra Paiva Araújo² (D) Janice Maria Borba Souzaㄹ (D), Liléia Gonçalves Diotaiuti (D), Raquel Aparecida Ferreira' (D)

'Grupo de pesquisas em Triatomíneos, Instituto René Rachou (IRR), Fundação Oswaldo Cruz (Fiocruz) - Belo Horizonte (MG), Brasil. ${ }^{2}$ Departamento de Ciências Básicas da Vida, Universidade Federal de Juiz de Fora - Governador Valadares (MG), Brasil.

${ }^{3}$ Secretaria Estadual de Saúde de Minas Gerais, Superintendência Regional de Divinópolis - Divinópolis (MG), Brasil.

Como citar: Rodrigues FCS, Souza ICA, Araújo AP, Souza JMB, Diotaiuti LG, Ferreira RA. Agentes comunitários de saúde: percepção sobre os serviços de saúde relacionados à doença de Chagas. Cad Saúde Colet, 2020;28(1):130-139. https://doi.org/10.1590/1414-462X202000280458

\section{Resumo}

Introdução: A doença de Chagas ainda é um importante problema de saúde pública na América Latina, apresentando alta prevalência, ampla distribuição geográfica e grande impacto social. Vários serviços relacionados à doença de Chagas são solucionados na Atenção Primária à Saúde (APS). Na Estratégia Saúde da Família (ESF), destaca-se o agente comunitário de saúde (ACS), profissional responsável pela mediação entre a comunidade e as unidades básicas de saúde (UBS). Objetivo: Apreender a percepção dos coordenadores da ESF e dos ACSs sobre o conhecimento a respeito da doença de Chagas e dos serviços de saúde relacionados nos municípios da microrregional de saúde de Itaúna, em Minas Gerais, Brasil. Método: Foram aplicados questionários semiestruturados aos ACSs e realizados grupos focais com os coordenadores da ESF. Resultados: Foi observado que, entre os profissionais, há ausência de informações básicas relacionadas à doença de Chagas; inexiste um protocolo/fluxograma de serviço; há uma ineficiência dos serviços de referência e contrarreferência; ocorre precariedade nas condições de trabalho; há ausência de capacitação dos profissionais e de ações de promoção à saúde nos municípios. Conclusão: Este é o primeiro estudo abordando esta temática: percepção dos ACSs sobre a doença de Chagas e seus serviços. Sugere-se a extensão deste estudo a outras regiões do Brasil.

Palavras-chave: doença de Chagas; agentes comunitários de saúde; atenção primária à saúde; serviços de saúde.

\begin{abstract}
Background: Chagas disease is still an important public health issue in Latin America, presenting high prevalence, broad geographical distribution and great social impact. Several services related to Chagas disease are solved in Primary Health Care (PHC). In the Family Health Strategy (FHS) it is highlighted the Community Health Agent (CHA), professional responsible for mediating between the community and units basics of health (UBH). Objective: To raise the perception of the coordinators of the FHS and the CHAs about the knowledge on Chagas disease and related health services in the municipalities of the micro-regional health of Itaúna, Minas Gerais, Brazil. Method: Semi-structured questionnaires were applied to the CHAs and focus groups were carried out with coordinators of the FHSs. Result: It was observed that among professionals there is no basic information related to Chagas' disease; the lack of a service
\end{abstract}

Trabalho realizado nos municípios que compõem a microrregional de saúde - Itaúna (MG), Brasil.

Correspondência: Raquel Aparecida Ferreira. E-mail: raquel.ferreira@fiocruz.br

Fonte de financiamento: FAPEMIG -Edital Programa de Pesquisa para o SUS (PPSUS) 14/2013, IRR/FIOCRUZ, SES/MG.

Conflito de interesses: nada a declarar.

Recebido em: Set. 13, 2018. Aprovado em: Abr. 11, 2019
Este é um artigo publicado em acesso aberto (Open Access) sob a licença Creative Commons Attribution, que permite uso, distribuição e reprodução em qualquer meio, sem restrições desde que o trabalho original seja corretamente citado. 
protocol/flowchart was noticed; inefficiency of reference and counter/reference services; precariousness in working conditions and training of professionals and absence of health promotion actions in municipalities. Conclusion: This is the first study addressing this theme: perception of professionals about Chagas disease and its services. It is suggested that this study be extended to other regions of Brazil.

Keywords: Chagas disease; community health agents; primary health care; health services.

\section{INTRODUÇÃO}

A doença de Chagas é causada pelo protozoário flagelado Trypanosoma cruzi, e sua principal forma de transmissão humana relatada nos últimos anos no Brasil é a transmissão oral' . Considerada uma doença negligenciada, é endêmica em 21 países e afeta, aproximadamente, 6-7 milhões de pessoas ${ }^{2}$, enquanto 90 milhões estão expostas ao risco de infecção ${ }^{3}$.

Em 1994, foi criado o Programa Saúde da Família (PSF), e, diante de sua amplitude, considerou-se que, mais que um programa, a saúde da família tratava-se de uma estratégia que impulsionaria o desenvolvimento da Atenção Primária à Saúde. A Estratégia Saúde da Família (ESF) se caracteriza por apresentar uma equipe multiprofissional composta de: médico generalista, enfermeiro, auxiliar/técnico de enfermagem e agente comunitário de saúde (ACS) - este considerado o mediador da comunidade com os profissionais e as unidades de saúde 4 . Os ACSs têm atuação na localidade em que residem, o que contribui para um melhor conhecimento da população, facilitando a identificação das situações vulneráveis e auxiliando na atuação em busca de alternativas para a melhoria das condições de saúde dos indivíduos, por meio de cadastros, visitas domiciliares e orientações às famílias 5 .

A Atenção Primária à Saúde (APS) é conhecida por ser a porta de entrada dos usuários ao Sistema Único de Saúde (SUS) ${ }^{6}$. Na APS, devem ser acompanhados os quadros clínicos não graves da doença de Chagas e também realizado o tratamento etiológico com benznidazol, quando indicado, para casos de doença de Chagas aguda ou crônica não grave ${ }^{7}$. Apesar de vários serviços relacionados à doença de Chagas serem solucionados na APS, não há na literatura nenhum estudo ligado ao tema. Dessa forma, o objetivo desta pesquisa foi avaliar a percepção dos ACSs e coordenadores da ESFs sobre aspectos referentes ao conhecimento acerca da doença de Chagas e serviços de saúde relacionados nos municípios da microrregional de saúde de Itaúna. Essa microrregional está situada em um dos locais de destaque no cunho histórico sobre a doença de Chagas e seu controle no Estado de Minas Gerais.

\section{MÉTODO}

Este estudo foi desenvolvido em Itaguara, Itatiaiuçu, Itaúna e Piracema, os quatro municípios que compõem a microrregional de saúde de Itaúna, a qual é pertencente à Superintendência Regional de Saúde (SRS) de Divinópolis. O inquérito sorológico nacional realizado entre 1970 e os primeiros anos de $1980^{8}$ apontou a seguinte prevalência da doença nos municípios da microrregional: Itaguara, 6,1\%; Itatiaiuçu, 23,5\%; Itaúna, 25,4\%; e Piracema, 4,1\%.

Primeiramente, foi utilizado um questionário semiestruturado composto de 14 questões, aplicado a 21 ACSs de Itaguara, 14 ACSs de Itatiaiuçu, 16 ACSs de Itaúna e 14 ACSs de Piracema, totalizando 65 ACSs.

O questionário continha dois eixos estruturais: doença de Chagas/profissionais de saúde; e serviços de saúde relacionados à doença de Chagas. O primeiro eixo continha nove questões que tratavam da percepção dos participantes sobre aspectos relacionados ao conhecimento dos profissionais de saúde dos municípios acerca da doença de Chagas. O segundo eixo continha cinco questões que tratavam da percepção dos participantes acerca do conhecimento dos profissionais de saúde sobre o fluxo de serviços relacionados à doença. Cada participante avaliou as questões e, de acordo com a sua percepção, marcou alternativa como: sim, não ou em branco.

Posteriormente, foram realizadas a análise dos questionários e a quantificação do percentual das respostas em função da percepção dos participantes, sendo efetuada a média das porcentagens relacionadas às respostas dos profissionais.

A partir da análise dos resultados dos questionários, foi elaborado um roteiro semiestruturado para realização de grupos focais com os coordenadores das ESFs dos 
municípios. O roteiro foi composto de 15 perguntas levantando à percepção dos participantes a respeito do conhecimento dos profissionais de saúde sobre a doença de Chagas e seus vetores, sobre o fluxo de serviços de saúde relacionados à doença e sobre as ações de promoção à saúde relativas à doença.

Foram realizados quatro grupos focais com os coordenadores das ESFs dos municípios. Os grupos focais foram gravados, tiveram os áudios transcritos e analisados por meio da técnica de análise de conteúdo?.

O estudo foi submetido ao Comitê de Ética em Pesquisa do Instituto René Rachou sob o número de certificado de apresentação de apreciação ética 53400616.8.0000.5091 e aprovado. Todos os participantes dos questionários e grupos focais assinaram o Termo de Consentimento Livre e Esclarecido (TCLE), concordando com a participação na pesquisa.

\section{RESULTADOS/ DISCUSSÃO}

\section{Percepção dos ACSs sobre aspectos relacionados ao conhecimento e aos serviços de saúde referentes à doença de Chagas}

Dos 179 ACSs convidados nos municípios, 65 (36\%) responderam ao questionário. A síntese das questões e as respectivas porcentagens estão descritas na Tabela 1. Primeiramente, $86,7 \%$ dos ACSs dos municípios afirmaram que não eram oferecidos cursos latualizações referentes à doença de Chagas (Tabela 1). Contraditoriamente, $75 \%$ dos participantes alegaram que o conhecimento dos profissionais de saúde relacionado à doença era satisfatório, e 60,7\% afirmaram que a equipe de saúde da família detinha conhecimento a respeito de sintomas clínicos da doença de Chagas (Tabela 1). Além disso, o percentual de profissionais que sabiam

Tabela 1. Porcentagem de respostas relacionadas à percepção dos agentes comunitários de saúde da microrregional de saúde de Itaúna sobre aspectos relacionados ao conhecimento sobre a doença Chagas

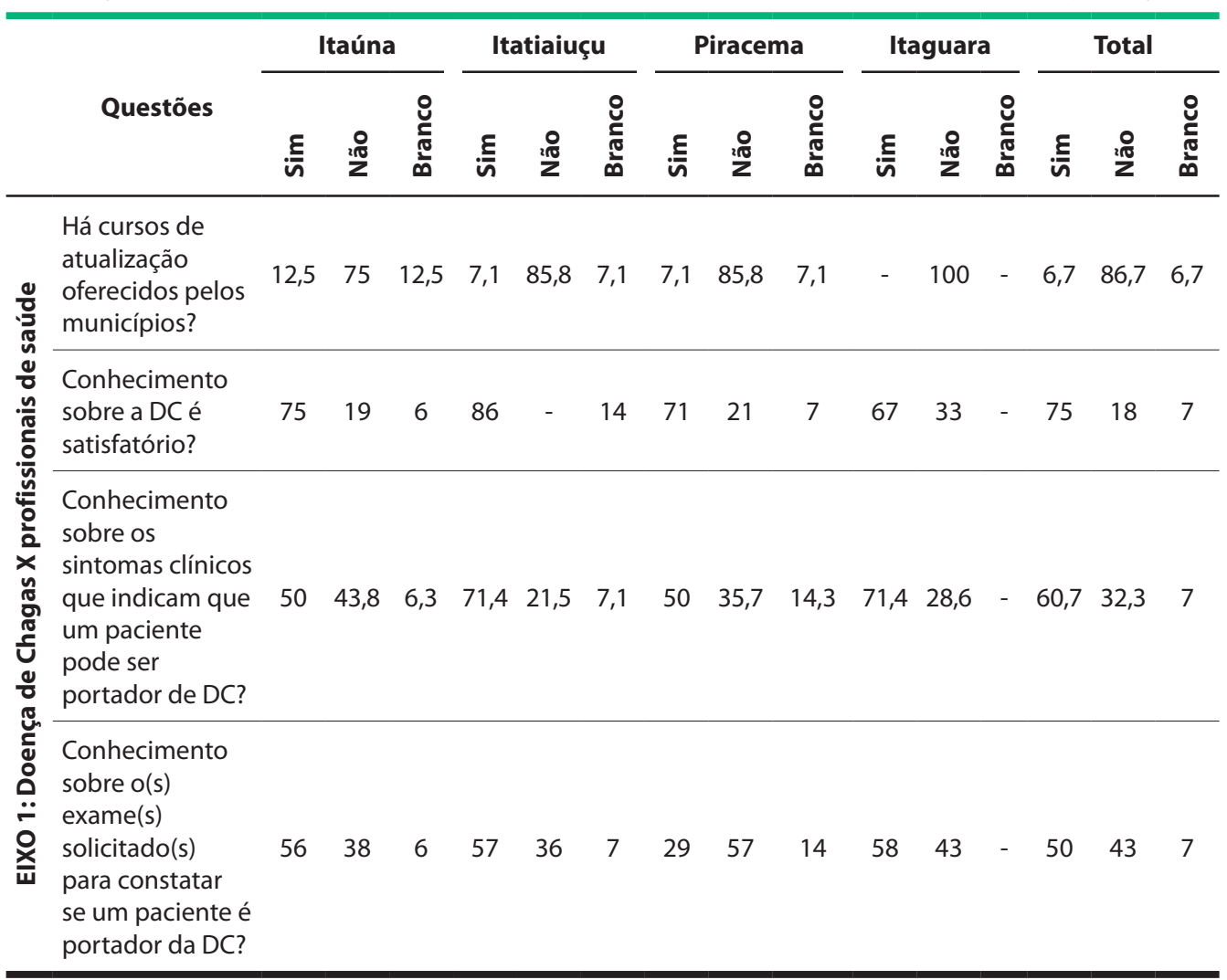


quais eram os exames diagnósticos da doença de Chagas foi superior a 50\% em quase todos os municípios, com exceção de Piracema (Tabela 1). Portanto, apesar de a maioria dos agentes afirmar que não havia cursos de capacitação relacionados à doença de Chagas voltados aos ACSs nos municípios, a maior parte deles acreditava ter um conhecimento satisfatório a respeito da doença.

Mais de $70 \%$ dos profissionais dos municípios, com exceção de $56,2 \%$ dos participantes de Itaúna, perceberam a existência de ações de promoção à saúde desenvolvidas nos municípios, e ações para a população exposta a fatores de risco de contrair a doença (Tabela 2). Além disso, $61 \%$ dos ACSs dos quatro municípios entendiam que havia intervenções sobre os determinantes sociais de saúde sendo realizadas nos municípios (Tabela 2). Esse resultado não está dentro do esperado, já que a maioria dos agentes reconhecia que não havia capacitação dos profissionais e que o controle da doença de Chagas continuava negligenciado 3 .

Tabela 2. Porcentagem de respostas relacionadas à percepção dos agentes comunitários de saúde da microrregional de saúde de Itaúna sobre os serviços relacionados à doença de Chagas

\begin{tabular}{|c|c|c|c|c|c|c|c|c|c|c|c|c|c|c|c|c|}
\hline & \multirow[b]{2}{*}{ Questões } & \multicolumn{3}{|c|}{ Itaúna } & \multicolumn{3}{|c|}{ Itatiaiuçu } & \multicolumn{3}{|c|}{ Piracema } & \multicolumn{3}{|c|}{ Itaguara } & \multicolumn{3}{|c|}{ Total } \\
\hline & & 站 & i̊ & 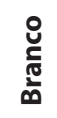 & 结 & io & 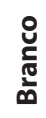 & 틀 & i̊ & 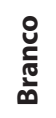 & 틀 & 㸾 & $\underset{\substack{\frac{0}{0} \\
\frac{0}{\infty}}}{ }$ & 站 & $\frac{0}{2 \pi 0}$ & 选 \\
\hline & $\begin{array}{l}\text { Há ações de } \\
\text { promoção } \\
\text { à saúde nos } \\
\text { municípios? }\end{array}$ & 43,8 & 56,2 & - & 78,6 & 21,4 & - & 71,4 & 28,6 & - & 71,4 & 28,6 & - & 66,3 & 33,7 & - \\
\hline & $\begin{array}{l}\text { Possui algum } \\
\text { tipo de } \\
\text { intervenção } \\
\text { sobre os } \\
\text { determinantes } \\
\text { sociais da } \\
\text { saúde? }\end{array}$ & 50 & 38 & 13 & 71 & 29 & - & 57 & 43 & - & 67 & 24 & 10 & 61 & 33 & 6 \\
\hline 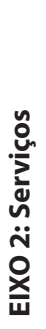 & $\begin{array}{l}\text { Detém ações } \\
\text { de prevenção } \\
\text { à saúde, para } \\
\text { a população } \\
\text { exposta a } \\
\text { fatores de } \\
\text { riscos de } \\
\text { contrair a } \\
\text { doença? }\end{array}$ & 43,8 & 56,2 & - & 71,4 & 28,6 & - & 64,3 & 35,7 & - & 66,7 & 33,3 & - & 62 & 38 & - \\
\hline & $\begin{array}{l}\text { Os } \\
\text { profissionais } \\
\text { dominam } \\
\text { todo o fluxo } \\
\text { de serviço } \\
\text { relacionado a } \\
\text { DC? }\end{array}$ & 38 & 56 & 6 & 57 & 43 & - & 64 & 29 & 7 & 29 & 71 & - & 47 & 50 & 3 \\
\hline & $\begin{array}{l}\text { Os } \\
\text { profissionais } \\
\text { sabem } \\
\text { para onde } \\
\text { encaminhar } \\
\text { o vetor } \\
\text { suspeito? }\end{array}$ & 81,2 & 12,5 & 6,3 & 71,4 & 28,6 & - & 85,8 & 7,1 & 7,1 & 66,7 & 33,3 & - & 76 & 21 & 3 \\
\hline
\end{tabular}


A maioria dos ACSs de Itaúna e de Itaguara entedia que os profissionais de saúde dos municípios desconheciam o fluxo de serviços relacionados à doença de Chagas, ao contrário de $57,1 \%$ dos ACSs de Itatiaiuçu e $64,3 \%$ de Piracema (Tabela 2). Em contradição, $76 \%$ dos ACSs, de todos os municípios, afirmaram que os profissionais de saúde dos municípios sabiam para onde encaminhar o inseto suspeito de ser transmissor do parasito causador da doença quando entregue pela população (Tabela 2).

Por meio desses resultados, foi verificada a existência de contradições e variações na percepção dos profissionais sobre algumas questões levantadas. Para esclarecer e melhorar a compreensão dessas diferentes concepções, foram realizados grupos focais com os coordenadores da APS das ESFs dos municípios.

\section{Percepção dos coordenadores das ESFs sobre aspectos relacionados ao conhecimento e aos serviços de saúde referentes à doença de Chagas.}

Foram trabalhadas as seguintes categorias: doença de Chagas; saúde; serviços de saúde; e profissionais de saúde.

Os coordenadores relataram baixo número de entrada de pacientes portadores do T. cruzi nas Unidades básicas de Saúde (UBSs) dos municípios, e ainda destacaram que a maioria deles era idosa. Essas afirmações foram apreendidas por meio das falas dos profissionais quando indagados sobre a existência de casos crônicos da doença nas comunidades assistidas pelas equipes de saúde da família. A seguir consta um exemplo dessas afirmações:

Eu tive, mas na época que eu tava trabalhando lá no Garcia. É um bairro muito próximo da zona rural, então, era um senhor já mais velho, com histórico já de.. de doença de Chagas e cardiopatia, consequência dela né? Então num [...] Foi só esse que eu me lembro mesmo (Coordenador 11).

Esses achados são interessantes e, ao mesmo tempo, intrigantes, uma vez que Minas Gerais é um dos Estados com maior prevalência da doença de Chagas no Brasil ${ }^{10}$. Além disso, a região centro-oeste é uma das mais endêmicas de Minas, já que a população da região conviveu com o Triatoma infestans, principal vetor do T. cruzi, colonizando seus domicílios e peridomicílios na década de $1940^{11}$. Logo, era esperado um maior número de portadores crônicos da doença utilizando os serviços da APS dos municípios. Porém, não se pode ignorar o fato de que grande parte dos portadores do parasito, possivelmente, está na fase indeterminada da doença ${ }^{12}$, ou seja, não apresenta sintomatologia e possivelmente não é diagnosticada. Essa é uma situação grave, que pode significar que essas pessoas não estão sendo acompanhadas clinicamente, conforme recomendado pelo II Consenso Brasileiro em Doença de Chagas ${ }^{12}$ e pelo PCDT ${ }^{7}$. Também é necessário ressaltar que o inquérito sorológico nos municípios está desatualizado. Outras possíveis explicações para o pequeno número de portadores da infecção pode ser o fato de estes não estarem necessitando de assistência médica ou relatando sintomas da doença durante as consultas médicas. Soma-se a esse fato a ausência de uma abordagem mais redundante dos profissionais de saúde durante a anamnese, o que, de certa forma, demonstra negligência do sistema de saúde pública em relação aos programas e ações de controle da doença de Chagas. Seguem falas que corroboram com essa ideia:

Então acho que não é comum as pessoas: eu sou chagásico na consulta, igual quando a pessoa é alérgica, tem outro problema. A eu tenho problema nas ritimia, ai quando, claro o profissional chega e faz uma boa anamnese vai perguntando, aí ele fala (Coordenador 12).

Então eu mesmo nunca perguntei na minha anamnese se a pessoa: A já teve [...] Foi picado por um barbeiro, cê tem alguém na família alguém com... Com doença Chagas? Então eu acho que o talvez é sensibilizar o profissional né, porque ás vezes [...] A gente pergunta muito em relação a hipertensão, diabetes, doenças que são muito comuns pra gente no dia a dia. (Coordenador 5). 
Diante das falas anteriores, é possível perceber que a falha no processo de identificação do portador crônico da doença, seja ele sintomático, seja de fase indeterminada, tem início na entrada do usuário nos serviços de saúde. Essa falha perpassa o acolhimento, momento em que o profissional deveria centrar a atenção no indivíduo, o que ressalta a importância de uma melhor abordagem e relacionamento interpessoal, uma vez que o paciente, ao procurar o estabelecimento de saúde, encontra-se mais fragilizado e, muitas vezes, tem dificuldades em relatar suas queixas/sintomas de forma clara. Além disso, essa falha na identificação do portador também está relacionada ao desconhecimento do profissional de saúde sobre a epidemiologia da doença, o que dificulta uma anamnese mais detalhada, envolvendo não somente o ser "indivíduo", mas englobando o ambiente em que esse indivíduo está inserido.

Ainda que existam no Brasil de 1,9 a 4,6 milhões de portadores crônicos da doença ${ }^{13-14}$, há relativamente poucos óbitos associados, o que, provavelmente, influencia o relaxamento de programas, ações, promoção e prevenção ${ }^{3}$, e até mesmo do próprio paciente. De fato, algumas falas mencionaram a ideia de que havia pacientes que não percebiam a doença de Chagas como um problema, conforme destacado a seguir:

Então é isso! Coloca assim, parece que não é um problema que eles têm. Eles não percebe, meu pai mesmo pelo menos não percebe isso como um problema pra ele. (Coordenador 11).

Nenhum profissional destacou a realização de atendimento a casos agudos, como demonstrado na fala seguinte:

[...] mas,... chegar assim com suspeita, de que houve comprometimento, que foi .. picado por um barbeiro, eu num peguei essa situação ainda não. (Coordenador 10).

A escassez de entrada de portadores da doença de Chagas na APS é uma realidade. Em contraponto, os profissionais relataram a existência de considerável número de vetores do parasito causador da doença entregues nas UBSs ou nos postos de informação de triatomíneos (PITs), conforme expresso na fala a seguir.

Tem vetor em q, positivo, mas não tem doente, não tem, nunca detectamos. (Coordenador 2).

Os PITs são locais escolhidos pela vigilância em saúde, nos quais a comunidade pode entregar insetos suspeitos de ser os vetores do T.cruzi. A população deveria conhecer a localização dos PITs em sua comunidade. Entretanto, muitos usuários parecem entregar esses insetos em UBSs, em PSFs ou aos ACSs, conforme a fala seguinte:

E os pacientes também, quando encontra, tipo, não só barbeiro, mas algum outro/vetor [...] Vetor assim, eles levam para o PSF. (Coordenador 9).

Ficou evidente a dificuldade dos profissionais em identificar os PITs pela terminologia utilizada (triatomíneos).

Se... se você for por Triatomíneo ninguém vai saber identificar... (Coordenador 1).

Acredita-se que a alteração do nome triatomíneos por outro nome popular do inseto, por exemplo, posto de informação de barbeiros ou posto de informação de bicudos, dependendo de como o inseto é conhecido na região, melhoraria a compreensão dos usuários e, consequentemente, a efetividade dos postos.

Em relação ao fluxo de acesso e ao atendimento dos pacientes portadores de Chagas, é observado nas falas que os profissionais evidenciaram ter esse conhecimento:

Se você tiver um caso mais grave, né? [...] Que já é uma pesquisa mais crônica, já encaminha, pra o cardiologista, né? o' [...] Mas o primeiro atendimento é feito normalmente aqui no PSF. (Coordenador 4). 
A Secretária de Vigilância da Saúde (SVS) elaborou um manual contendo os procedimentos e o conjunto de ações que orientam os profissionais a como proceder com um portador de doença de Chagas ${ }^{15}$. No entanto, os serviços de referência e contrarreferência, compreendidos como encaminhamentos recíprocos de pacientes da UBS para o Centro de Especialidades Médicas (CEM) e outros níveis de complexidade de serviços, não têm sido executados como preconizado pela lei orgânica de saúde. Isso foi reproduzido nas falas de alguns participantes:

[...] aí se a gente não for atrás do paciente a gente fica sem referência/Muitas vezes o paciente ele não entende nada! Se perguntar ele: nun sei... Não sabe nada!...O quê que o médico te mandou? Pra qual que é o médico? [...] A gente fica perdido assim no meio, muitas vezes, num sei... num tem interação. [...] Num tem essa interação que a gente né acabou aqui, é isso que falta muitas vezes, mas ai a gente não sabe se foi pra um cardiologista, o quê que foi, qual que é a especialidade, que é que ele vai, a gente fica perdido. (Coordenador 4).

A ausência dos serviços integrados de referência/contrarreferência de exames de pacientes e de vetores foi uma fala recorrente dos entrevistados. A regulação da referência e contrarreferência ${ }^{16}$ é um serviço habitualmente do coordenador dos serviços de saúde e depende totalmente dele para sua efetivação nos processos de planejamento, oferta dos serviços para população, elaboração de metas, monitoramento e avaliação.

Interessantemente, nos municípios os profissionais perceberam que havia uma boa integração e comunicação entre a APS e o setor de epidemiologia, conforme destacado na fala a seguir:

Vetor/Vetor assim, eles levam para o PSF sabe. Levam num vidrinho e a gente anota endereço, e tudo e passa pra esses agentes (de endemias, grifo nosso). (Coordenador 8).

As condições precárias de habitação, saneamento, educação e pouco acesso às redes de serviços de saúde da população influenciam diretamente a transmissão da doença de Chagas ${ }^{17}$. A promoção à saúde remete ao pensamento de que o indivíduo também é responsável por sua saúde, diminuindo, assim, a responsabilidade do Estado, o qual, entretanto, continua com o dever na criaçãolelaboração de políticas públicas e ações orientadas para a qualidade de vida da população ${ }^{18}$. Quando os coordenadores foram questionados sobre a existência de ações de promoção à saúde relacionada à doença de Chagas, foi explicitada, em vários momentos, a ausência delas:

Uma dificuldade que eu acho que também pode ser, porque apesar de cada PSF uma realidade, uma demanda específica, a gente falta recurso humano, porque nós somos uma enfermeira da coordenação e uma técnica. Então, às vezes, fica muita assistência, a gente fica muito na assistência, burocracia que só aumenta, e fica a desejar essa parte de promoção de saúde, de envolver a equipe, às vezes envolver usuário, a gente não tem como avisar, não é que num é que num tem. (Coordenador 4).

A Conferência Internacional de Promoção à Saúde, realizada no Canadá19, divulgou a Carta de Ottawa, documento que instituiu a promoção à saúde como: "processo de preparação do estado, comunidade e indivíduos, para o aconselhamento, orientação e educação individual e coletiva para atuarem na mudança da qualidade de vida". Isso vai contra a realidade apontada pelos coordenadores nos municípios. As falas dos participantes expressaram várias deficiências nos municípios em relação às ações de promoção à saúde, as quais são atribuídas à sobrecarga de trabalho e à falta de recursos humanos e ações imediatistas voltadas para a cura, e não para a promoção. Contraditoriamente, os ACSs consideraram que nos municípios existiam ações de promoção à saúde relacionadas à doença Chagas. Uma possível explicação seria a de que esses profissionais possuem um conceito equivocado em relação ao termo "promoção à saúde", fato que não é raro.

No que se refere às políticas e ações de saúde, alguns coordenadores afirmaram que a inexistência de tais ações se devia ao fato de a doença de Chagas não apresentar alto número de 
casos, como foi no passado e conforme discutido anteriormente. Segue um trecho mostrando essas percepções:

Quando eu fui gestora da outra vez, eu acho que a coisa era mais feia... mais preocupante, a gente trabalhava mais em cima da doença [...] já trabalhou muito em cima doença de Chagas aqui, em 2005, 2006, a gente trabalhou, muito em cima da doença de Chagas, sabe?! Não que tivesse a doença, mais a gente trabal hava em cima, muito disso... depois foi... foi graças a Deus, foi melhorando... melhorando... hoje, cê vê que já tivemos mais de $30 . . .40$ casas que deu positivo! Hoje 4! Né...já... os focos já... a gente conseguiu, ta conseguindo acabar. Num acaba, ne gente!? Num vai zera, mais é... a gente já teve casos e casos... muitos casos... Hoje é eu sou tranquila nessa parte de Chagas. (Coordenador 1).

É sugerido que, na ausência de casos, a doença de Chagas, aparentemente, perde prioridade orçamentária e política para outras endemias com maior número de casos, tais como dengue e Zika. Ainda, além de um melhor prognóstico financeiro e político, essas endemias também são mais exploradas e evidenciadas pelas mídias e até mesmo pelas instituições de saúde. Também não pode ser desconsiderado o fato de que os casos da doença de Chagas sempre foram difíceis de diagnosticar, uma vez que a doença apresenta um grande número de casos assintomáticos, tanto na fase aguda quanto na fase crônica. Essa dificuldade ocorreu mesmo quando a transmissão do parasito era mais intensa. Também foi observado que a maioria dos portadores do parasito não relatou a doença para os profissionais, demonstrando negligência em relação à doença. Isso esteve refletido nas falas dos profissionais ao informar a ausência de conhecimento ou informação da doença pelos usuários:

Eu pego como exemplo: Meu pai é chagásico, não é desse município, não mora aqui, e já tem oitenta anos, diagnosticou Chagas na fase adulta dele (adulto mais jovem né) e sempre [...] Ás vezes quando meu pai vai no profissional médico, quando vai fazer alguma revisão no card [...] Até comenta. Mais é comum, hoje quando ele vai, vai no outro [...] Então acho que não é comum as pessoas: Eu sou chagásico na consulta, igual quando a pessoa é alérgica, tem outro problema. A eu tenho problema nas ritimia, ai quando, claro o profissional chega e faz uma boa anamnese vai perguntando, ai ele fala. (Coordenador 11).

No Brasil no ano de 1992, iniciou-se o Programa de Agentes Comunitários de Saúde (PACS), e, em 1999, foram definidas as seguintes atribuições para os ACSs: práticas centradas na saúde da família, como prevenção e promoção à saúde, por meio de ações educacionais nos domicílios e nas comunidades, além de ações individuais e coletivas ${ }^{20}$. Sua função como mediadores entre a população e os profissionais de saúde, por serem moradores da área de abrangência em que atuam e por conhecerem a população e os fatores de riscos existentes, foi refletida na fala dos gestores, quando questionados sobre a recepção dos ACSs na residência dos usuários do SUS:

É... o que eu acabei de te falar: os agentes de saúde não têm problema não! Todo mundo aceita bem. Que vai marca consulta, que vai marca dentista, que vai fazer isso... vai fazer aquilo. (Coordenador 1).

Foi relatada ausência de capacitação dos ACSs relacionada a doença de Chagas, o que impacta diretamente na falta de atenção despendida à doença nos municípios e, consequentemente, expõe a população a saberes populares, crenças e senso comum dos ACSs sobre aspectos da doença. De fato, entre as recomendações do Ministério da Saúde está a capacitação de profissionais de saúde para informar aos familiares e aos demais interessados as características clínico-epidemiológicas da doença de Chagas. A falta de um programa de capacitações ou educação continuada desses profissionais não é algo restrito aos municípios participantes do estudo, mas um problema que vem ocorrendo em grande parte dos municípios brasileiros, como citado em estudos realizados em Araçatuba, interior de São Paulo³. 


\section{CONSIDERAÇÕES FINAIS}

A relação construída entre os pesquisadores e os participantes da pesquisa se estabeleceu como uma estratégia para compreender a percepção dos ACSs dos municípios da microrregional de Itaúna e de seus coordenadores sobre os serviços de saúde relacionados à doença de Chagas, tema ainda não explorado na literatura.

Todos os portadores do T. cruzi que procuram assistência na APS dos municípios são casos crônicos. Além disso, grande parte dos usuários do serviço não acredita que a doença de Chagas ainda exista, sobretudo aqueles mais jovens. É possível perceber que a doença é extremamente negligenciada e que há uma desarticulação das ações de controle vetorial, além de falta de capacitação dos profissionais. Uma das causas apontadas para a negligência em relação à doença de Chagas é o baixo número de casos conhecidos da doença atualmente no Brasil e, particularmente, na região. Diante da extrema relevância do conhecimento dos casos crônicos pela vigilância epidemiológica, é sugerido, assim como o preconizado pelo Ministério da Saúde ${ }^{7}$, que a notificação compulsória dos casos crônicos da doença deva ser considerada, evidenciando o número de casos reais da doença no país.

Os coordenadores afirmaram que o fluxo de serviços relacionados à doença de Chagas é seguido pelos profissionais de saúde. Entretanto, os serviços de referência e contrarreferência dos resultados de exames de vetores e de pacientes não funcionam adequadamente em nenhum dos municípios. Além disso, foi constatado que os PITs existem e funcionam na região, mas é necessário adequar a terminologia adotada para melhorar a compreensão dos usuários e até mesmo dos profissionais de saúde. Também são recomendadas ações de divulgação e educação em saúde, melhorando o envolvimento da comunidade e a compreensão de aspectos do serviço e até mesmo da terminologia empregada. Ainda, é importante considerar as amplas e diversas ações desenvolvidas pelas associações de pessoas afetadas pela doença de Chagas, que auxiliam na divulgação de informações sobre a doença a seus familiares e sociedade como um todo 7 .

Por meio dos resultados deste estudo é evidenciada a existência de lacunas, bem como o desconhecimento dos profissionais sobre os serviços da APS relacionados à doença de Chagas nos municípios. Nesse sentido, o Ministério da Saúde ${ }^{7}$ também destaca a existência de incertezas a respeito do manejo da doença de Chagas, particularmente na APS. Dessa forma, é sugerido que os achados obtidos neste trabalho possam ser expandidos a outras regiões do Brasil, uma vez que hoje não existe em todo território nacional um programa de controle da doença que assegure efetividade nas ações de prevenção e controle. A partir da Portaria no 1.399, de 1999, a responsabilidade por essas ações foi repassada aos municípios e Estados. Isso resultou em perdas de ações de controle e provavelmente na desmobilização do trabalho participativo ${ }^{21}$.

É indispensável a existência de um protocolo/fluxograma que organize os serviços, além de ações de promoção à saúde e capacitação permanente dos profissionais para apoiar, organizar e agilizar as atividades e os serviços de saúde. Também é preconizado que ações de melhoria em educação e saúde da população e dos profissionais devam ser resgatadas nos municípios mediante ações de extensão universitária no Brasil, contribuindo para a prevenção e o controle da doença de Chagas e para a promoção da saúde dos portadores, em qualquer estágio clínico da doença crônica. Finalmente, com a implementação do protocolo clínico e das diretrizes terapêuticas da doença de Chagas $^{7}$, é esperado que sejam garantidos o acesso e a atenção ao portador do T.cruzi.

\section{AGRADECIMENTOS}

Agradecemos às agências e às instituições financiadoras e/ou às apoiadoras do trabalho: FAPEMIG, IRR/Fiocruz, SES/MG, Secretarias Municipais de Saúde de Piracema, Itaguara, Itaúna e Itatiaiuçu. Também agradecemos aos profissionais de saúde dos municípios da microrregional de saúde de Itaúna pela disponibilidade e pela atenção 


\section{REFERÊNCIAS}

1. Brasil. Ministério da Saúde. Secretaria de Vigilância em Saúde. Doença de Chagas aguda no Brasil: série histórica de 2000 a 2013. Bol Epidemiol. 2015;46(21):1-9.

2. Organização Mundial de Saúde. Doença de Chagas [Internet]. Genebra: OMS; 2008 [citado em 2018 mai 14]. Disponível em: http://www.who.int/es/news-room/fact-sheets/detail/chagas-disease-(americantrypanosomiasis)

3. Silveira A. Situação do controle da transmissão vetorial da doença de Chagas nas Américas. Cad. Saúde Pública, 2000;16(2 Suppl):35-42

4. Escorel S, Giovanella L, Magalhães de Mendonça MH, Castro Maia Senna M. O Programa de Saúde da Família e a construção de um novo modelo para a atenção básica no Brasil. Rev Panam Salud Publica. 2007;21(2-3):164-76. http://dx.doi.org/10.1590/S1020-49892007000200011. PMid:17565803.

5. Nunes MDO, Trad LB, Almeida BA, Homem CR, Melo MCIC. O agente comunitário de saúde: construção da identidade desse personagem híbrido e polifônico. Cad Saude Publica. 2002;18(6):1639-46. http:// dx.doi.org/10.1590/S0102-311X2002000600018.

6. Brasil. Ministério da Saúde. Secretaria de Atenção à Saúde Departamento de Atenção Básica. Política Nacional de Atenção Básica. Série Pactos pela Saúde. 4. ed. [Internet]. Brasília; 2006 [citado em 2017 ago 2]. (Série E. Legislação de Saúde). Disponível em: http://bvsms.saude.gov.br/bvs/publicacoes/ pacto_saude_v4_4ed.pdf

7. Brasil. Ministério da Saúde. Portaria $n^{\circ}$ 57, de 30 de outubro de 2018. Protocolo Clínicas e diretrizes terapêuticas da doença de Chagas no âmbito do Sistema Único de Saúde - SUS. Diário Oficial da União [Internet], Brasília, 31 de outubro de 2018; Seção 1. p. 41 [citado em 2018 jan 24]. Disponível em: http:// conitec.gov.br/images/PCDT_Doenca_de_Chagas.pdf

8. Camargo ME, Silva GR, Castilho EA, Silveira AC. Inquérito sorológico da prevalência de infecção chagásica no Brasil, 1975/1980. Rev Inst Med Trop São Paulo. 1984;26(4):192-20. http://dx.doi.org/10.1590/S003646651984000400003. PMid:6441232.

9. Bardin L. Análise de conteúdo. Lisboa: Edições 70; 2009.

10. Villela MM, Souza JMBD, Melo VDP, Dias JCP. Vigilância epidemiológica da doença de Chagas em programa descentralizado: avaliação de conhecimentos e práticas de agentes municipais em região endêmica de Minas Gerais, Brasil. Cad Saude Publica. 2007;23(10):2428-38. http://dx.doi.org/10.1590/S0102311X2007001000018. PMid:17891303.

11. Dias E. Profilaxia da doença de Chagas. Resumo das principais atividades do Centro de Estudos do Instituto Oswaldo Cruz em Bambuí, MG. Brasil-Médico. 1946;60(18):161-63.

12. Dias JCP, Machado EM, Borges EC, Moreira EF, Gontijo C, Azeredo BV. Chagas' disease in Lassance, Minas Gerais State: Clinical-epidemiological re-evaluation ninety years after the discovery by Carlos Chagas. Rev Soc Bras Med Trop. 2002;35(2):167-76. http://dx.doi.org/10.1590/S0037-86822002000200007. PMid:12011926.

13. Hotez PJ, Fujiwara RT. Brazil's neglected tropical diseases: An overview and a report card. Microbes Infect. 2014;16(8):601-6.

14. Martins-Melo FR, Ramos AN Jr, Alencar CH, Heukelbach J. Prevalence of Chagas disease in Brazil. Acta Trop. 2014;130:167-74. http://dx.doi.org/10.1016/j.actatropica.2013.10.002. PMid:24139912.

15. Brasil. Ministério da Saúde. Secretaria Nacional de Vigilância em Saúde. Sistema de Notificação de Agravos de Notificação. Doença de Chagas Aguda: Manual prático de subsídio à Notificação Obrigatória no SINAN. Brasília: Ministério da Saúde; 2004. p. 1-20.

16. Serra CG, Rodrigues PHA. Avaliação da referência e contrarreferência no Programa Saúde da Família na Região Metropolitana do Rio de Janeiro (RJ, Brasil). Cien Saude Colet. 2010;15(3 Suppl):3579-86. http:// dx.doi.org/10.1590/S1413-81232010000900033.

17. Dias JCP, Ramos AN Jr, Gontijo ED, Luquetti A, Shikanai-Yasuda MA, Coura JR, et al. II Consenso brasileiro em doença de Chagas, 2015. Epidemiol Serv Saúde. 2016;25(N. esp):7-86

18. Czeresnia D. O conceito de saúde e a diferença entre prevenção e promoção. In: Czeresnia D, Freitas CM. Promoção da saúde: conceitos, reflexões, tendências. Rio de Janeiro: Fiocruz; 2003. p. 39-54.

19. Organização Mundial de Saúde. Primeira conferência internacional sobre promoção da saúde [Internet] Genebra: OMS; 1986.

20. Bornstein VJ, Stotz EN. Concepções que integram a formação e o processo de trabalho dos agentes comunitários de saúde: uma revisão da literatura. Ciênc Saúde Coletiva. 2008; 13(1):259-268.

21. Villela MM, Souza JB, Mello VP, Azeredo BVDM, Dias JCP. Vigilância entomológica da doença de Chagas na região centro-oeste de Minas Gerais, Brasil, entre os anos de 2000 e 2003. Cad Saude Publica. 2005;21(3):878-86. http://dx.doi.org/10.1590/S0102-311X2005000300022. PMid:15868046. 